\title{
Pleomorphic Adenoma of Pterygomaxillary Space: A Rare Case Report
}

\author{
Lokesh K Penubarthi ${ }^{1}$, Rizvi Ali ${ }^{2}$
}

\begin{abstract}
Background: Pleomorphic adenoma (PA) is a benign tumor most common in major salivary glands. PA of pterygomaxillary space (PMS) is rare. Only one case of PA arising from the pterygopalatine fossa (PPF) was reported. We are presenting a second case, its presentation and surgical management, which is different and less morbid from the recently described reports.

Case description: A 40-year-old female presented with ill-defined right cheek swelling for the last 5 years. On evaluation, there was a firm mass in the PMS, which was diagnosed as PA. Surgical excision was planned with sublabial incision combined with lip split, which could entirely reach, delineate, and remove the tumor in toto. The patient has recovered well and was discharged on the 3rd day.

Conclusions: Pleomorphic adenomas of PMS present late due to their anatomical location and indolent course. Contrast-enhanced computed tomography scan is an important diagnostic tool. Fine-needle aspiration cytology from the buccal aspect reveals the probable histopathology. Sublabial incision with lip split is more useful in excising the moderate-sized tumors.

Keywords: Infratemporal fossa, Lip split, Pleomorphic adenoma, Pterygomaxillary space, Sublabial approach.

International Journal of Head and Neck Surgery (2020): 10.5005/jp-journals-10001-1355
\end{abstract}

\section{INTRODUCTION}

Pleomorphic adenoma (PA) is a benign neoplasm, which is common in major salivary glands. Some rare sites that were reported include pharynx, larynx, and nasal cavity. ${ }^{1}$ Till date, only one case of PA of pterygopalatine fossa (PPF) has been reported. ${ }^{2}$ Here, we are presenting a second case of PA of pterygomaxillary space (PMS), which extends into the infratemporal fossa (ITF) and present as an ill-defined cheek swelling, its radiology, and surgical technique that are different and less morbid than the previously described reports.

\section{Case Description}

A 40-year-old female patient reported to our specialty with swelling and heaviness in the right cheek for the last 5 years (Fig. 1). Physical examination was unremarkable except for an ill-defined swelling in the right buccal space opposite to molars obscuring the upper gingivolabial sulcus. The swelling was firm on palpation and restrictedly mobile. Contrast-enhanced computed tomography (CECT) revealed a well-defined minimally enhancing heterogeneous soft tissue lesion with cystic component and central calcifications. The posterolateral wall of maxilla was compressed and remodeled due to pressure by tumor (Fig. 2). There were well-maintained planes between the surrounding structures. Fine-needle aspiration cytology (FNAC) showed features of epithelial neoplasm.

Surgery was performed by a sublabial incision with lip split. Sublabial incision was made up to last molar that was combined with lip split (Fig. 3). Tumor was delineated from the surrounding musculature and soft tissue by blunt dissection. There was a welldefined gritty tumor (Fig. 4) filling the ITF posteriorly extending and firmly adhering the base of lateral pterygoid. The tumor was delineated from lateral pterygoid, masseter muscles and removed in toto. Histopathology revealed tumor tissues showing sheets, clusters, and ducts of benign epithelial cells and myoepithelial cells. Within the tissues, myxoid areas are noted (Fig. 5). There
1,2Department of ENT and Head-Neck Surgery, Narayana Medical College, Nellore, Andhra Pradesh, India

Corresponding Author: Lokesh K Penubarthi, Department of ENT and Head-Neck Surgery, Narayana Medical College, Nellore, Andhra Pradesh, India, Phone: +91 8008020809, e-mail: lokesh86p@gmail.com How to cite this article: Penubarthi LK, Ali R. Pleomorphic Adenoma of Pterygomaxillary Space: A Rare Case Report. Int J Head Neck Surg 2020;11(2):36-38.

Source of support: Nil

Conflict of interest: None

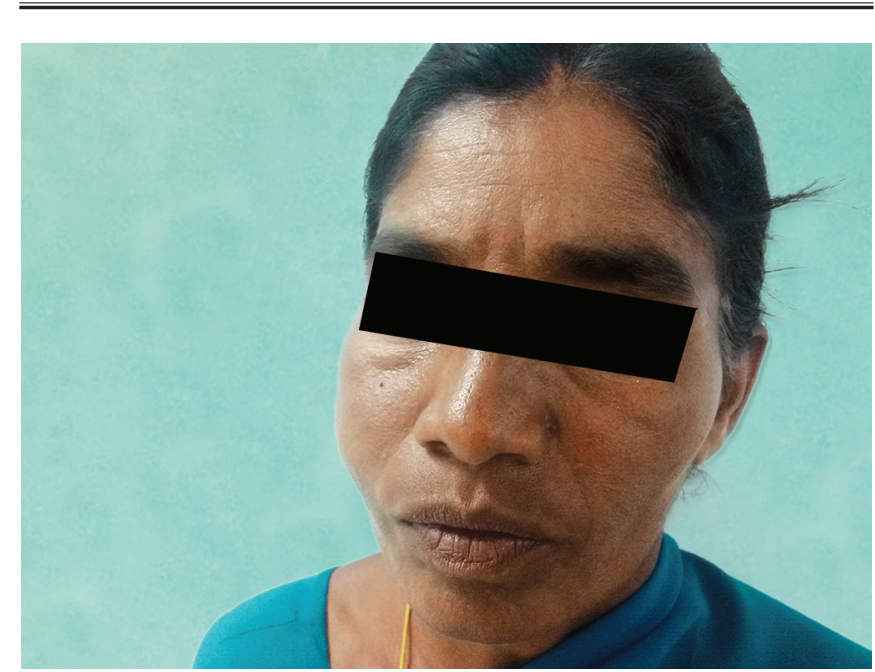

Fig. 1: Clinical photograph of the patient showing mild right cheek swelling

was no evidence of malignancy. Features were suggestive of PA. Postoperatively, the patient recovered soon and discharged on the 4th postoperative day. The patient was under follow-up for the last 1 year without any evidence of recurrence. 

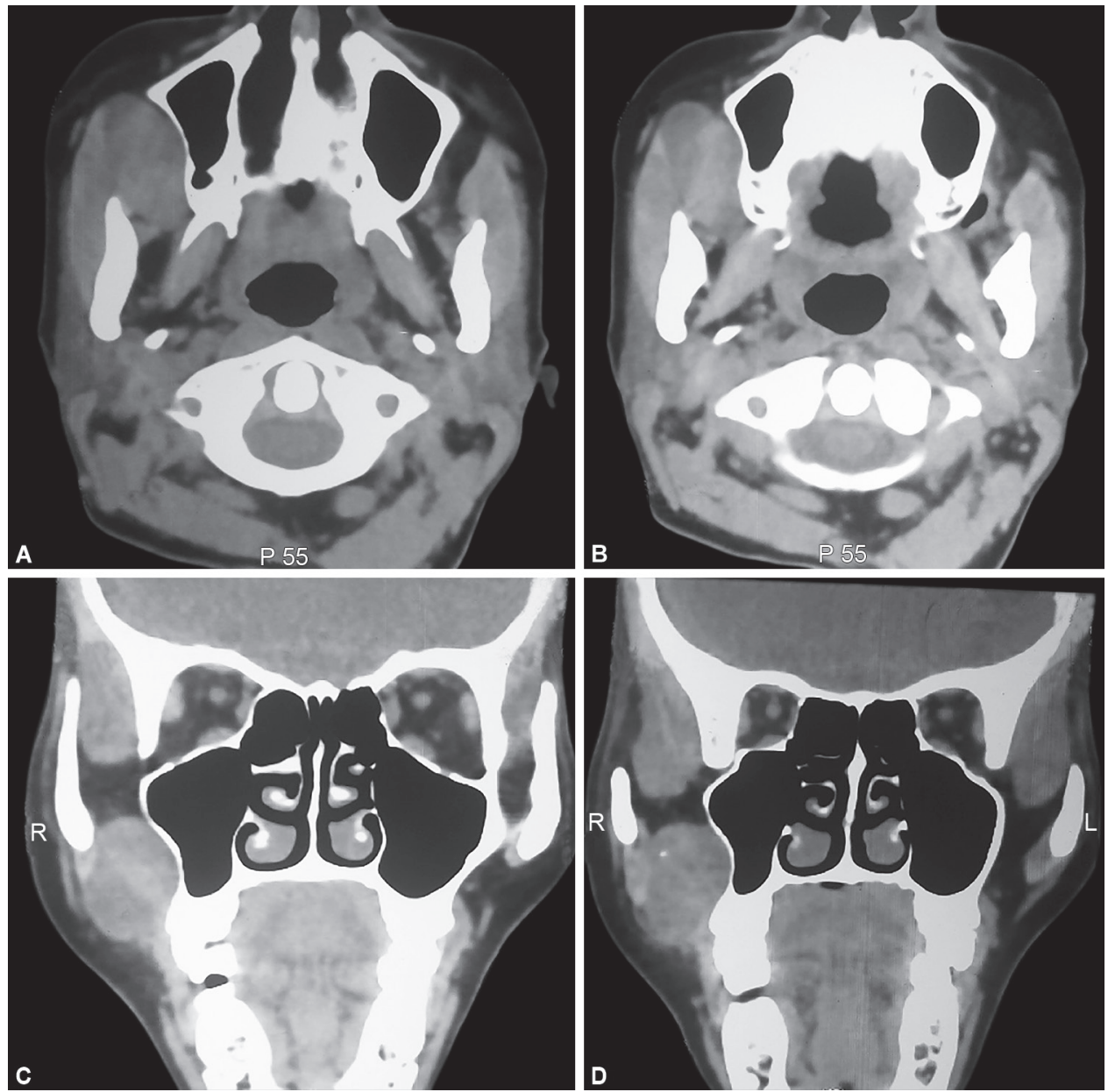

Figs 2A to D: Axial and coronal views of contrast-enhanced CT scan depicting the tumor location and extent

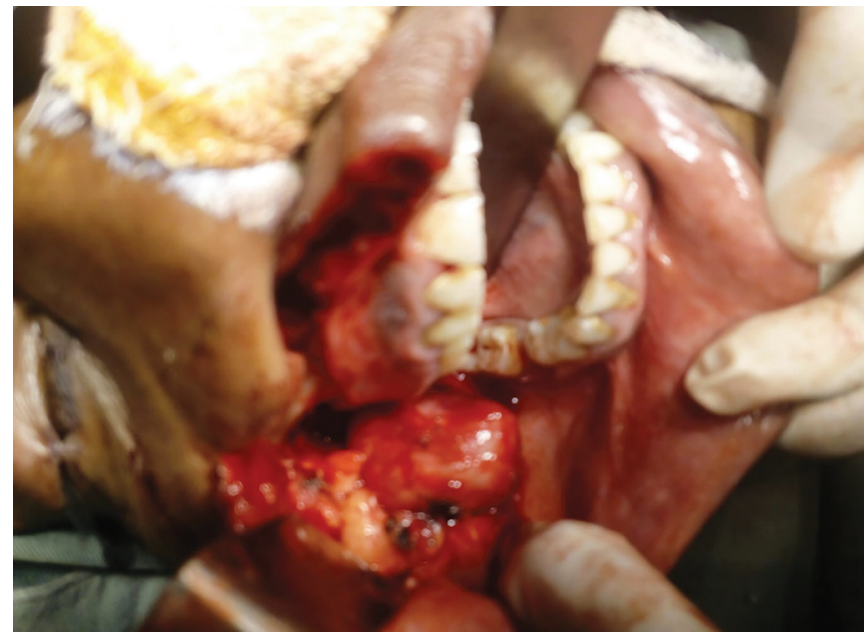

Fig. 3: Well-delineated tumor in the infratemporal fossa accessed through sublabial incision combined with lip split

\section{Discussion}

PMS is a potential three-dimensional space that lies between ITF and PPF. Tumors confined to these spaces are very rare. Commonly occurring tumors in this space are nasopharyngeal carcinomas, juvenile angiofibroma, and adenoid cystic carcinoma. ${ }^{2}$ Pleomorphic

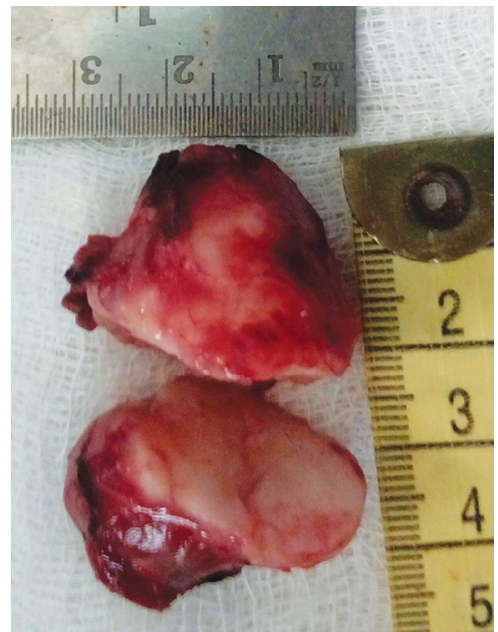

Fig. 4: Well-defined gritty tumor excised in toto

adenoma of PPF was reported by Kanazawa et al. ${ }^{3}$ Our case is the second case involving this region to the best of our knowledge.

Various hypotheses have been proposed regarding the origin of salivary tissue at abnormal locations. Ferlito et al. ${ }^{4}$ noticed heterotopic salivary tissue at various sites including pituitary, sternoclavicular joint, external ear canal, etc. All these heterotopic tissues have a potential for development of PA. Due to their 


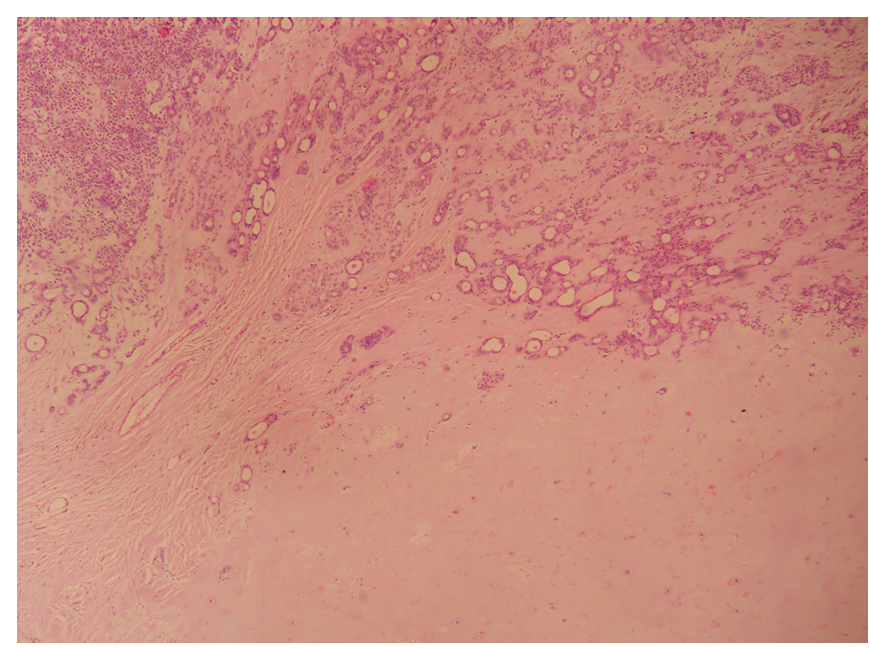

Fig. 5: Histopathology showing sheets, clusters, and ducts of benign epithelial cells and myoepithelial cells

anatomical location, benign tumors of this region present late $e^{5}$ as noticed in our case.

The CECT is an important diagnostic tool in determining the location, extent, and spread of the tumor and gives an idea about the adjacent structures. If the tumor is well enhancing, MR angiography is more helpful. In the present case, lesion was found to heterogeneously enhance with solid and cystic areas, so FNAC was performed which revealed an epithelial malignancy.

Surgical excision is the proven modality of treatment for PAs. PMS is a difficult area to reach as it is bounded anteriorly by the posterior wall of maxilla, posteriorly by the base of the greater wing of sphenoid, medially by palatine bone and lateral pterygoid plate, and superiorly by greater wing of sphenoid. Various approaches like preauricular transzygomatic, transmaxillary, transfacial, sublabial, and a combination of these have been described in previous reports. Kanazawa et al. ${ }^{3}$ and Tarik $^{6}$ used the transmaxillary approach through the anterior wall of maxilla to reach PPF and infratemporal space, respectively. Jayanti et al. ${ }^{5}$ used preauricular incision along with zygomatic osteotomy to reach infratemporal space. Our surgical technique is unique from these reports as no bony framework was disturbed and no osteotomies were used. Sublabial incision from incisors to last molars combined with lip split has given a good access to reach the site of lesion. All the surrounding structures are very well identified, delineated, and separated from the tumor. Even adequate hemostasis was achieved through this approach.

The patient recovered well, oral feeds were allowed 6 hours after surgery, and the patient was discharged on the 3rd day in our case. We attribute this early recovery to the less morbid approach we choose for this case.

\section{Conclusion}

Pleomorphic adenoma of PMS is very rare. Late presentation is due to indolent course and a hidden anatomical location. Contrastenhanced CT scan is an important tool in management. The FNAC from the buccal aspect of the tumor most of the times reveals the probable histopathology. Surgical excision through the sublabial incision combined with partial lip split is useful for moderate-sized tumors with limited extension without any need for disturbing a bony framework. A close follow-up for long duration is required as PAs are more prone to recurrence.

\section{References}

1. Suzuki K, Moribe K, et al. A rare case of pleomorphic adenoma of lateral wall of nasal cavity - with special reference of statistical observation of pleomorphic adenoma of nasal cavity in Japan. Nihon Jibiinkoka Gakkai Kaiho 1990;93(5):740-745. DOI: 10.3950/ jibiinkoka.93.740.

2. Llorente JL, Nazar G, et al. Subtemporal-preauricular approach in the management of infratemporal and nasopharyngeal tumours. J Otolaryngol 2006;35:173-179.

3. Kanazawa $\mathrm{T}$, Nishino $\mathrm{H}$, et al. Pleomorphic adenoma of the pterygopalatine fossa: a case report. Eur Arch Otorhinolaryngol 2000;257(8):433-435.

4. Ferlito A, Baldan M, et al. Implantation of parotid pleomorphic adenoma in the upper neck. ORL J Otorhinolaryngol Relat Spec 1991;53(3):165-176. DOI: 10.1159/000276212.

5. Jayanti K, Karthikeyan R, et al. Pleomorphic Adenoma in the Infratemporal Space: The First Case Report. Head and Neck Pathol 2007;1:173-177. DOI: 10.1007/s12105-007-0036-z.

6. El-Hadi T, Oujilal A, et al. Plemorphic Adenoma of the Infratemporal Space: A New Case Report. Int J Otolaryngol 2009;2009:529350. DOI: $10.1155 / 2009 / 529350$. 